\title{
Transmission and absorption properties of two-dimensional metallic photonic-band-gap materials
}

\author{
Lie-Ming Li, ${ }^{*}$ Zhao-Qing Zhang, and Xiangdong Zhang \\ Department of Physics, Hong Kong University of Science and Technology, Clear Water Bay, Kowloon, Hong Kong, China
}

(Received 21 July 1998)

\begin{abstract}
Using the multiple-scattering method, we study systematically the transmission and absorption properties of two-dimensional (2D) metallic photonic-band-gap (PBG) materials for a wide range of frequency and structure scales by adopting a realistic dielectric constant for metal cylinders. For both $S$ and $P$ waves, analytic expressions for the absorption power of a single metal cylinder under an arbitrary external field have been derived to explain the absorption behaviors of metallic PBG materials in a coherent way. The discussions and conclusions presented here can be generalized to 3D systems as well. [S0163-1829(98)00447-0]
\end{abstract}

\section{INTRODUCTION}

Photonic-band-gap (PBG) materials have been under intensive study during the past few years. ${ }^{1,2}$ The existence of gaps can have an important impact both in science and technology. Although most of the studies in the past were done for the positive frequency-independent dielectric PBG materials, there has been increasing interest in metallic PBG materials recently due to the existence of large full gaps facilitated by the large negative real part of the dielectric constant in the metallic component. ${ }^{3-9}$ In frequency-independent dielectric PBG materials, the only relevant variable in the system is the dimensionless frequency $f a / c$, where $f, a$, and $c$ are, respectively, the frequency, lattice constant, and the speed of light in the vacuum. However, in metallic PBG materials, due to the existence of a large frequencydependent imaginary part of the dielectric constant in the metal component, the scaling, which holds in the dielectric case, becomes invalid in the metallic case. Scaling applies only if a perfect conductor is assumed for the metal component. ${ }^{4,6,7}$ The interesting question is how the presence of absorption affects the position and quality of a gap.

In this work, by using the multiple-scattering method, we study systematically the transmission and absorption behaviors of two-dimensional (2D) metallic PBG materials for a wide range of frequency and structure scales. By adopting a realistic dielectric constant for metal cylinders, we find from the transmission results that, as far as the gap properties are concerned, scaling holds to a good approximation even in the presence of a large imaginary part in the dielectric constant. However, we find significant shifts in the gap positions if we ignore the imaginary part of the dielectric constant for metal cylinders. The absorption behaviors of metallic PBG materials have been studied in Ref. 5 by using the transfer-matrix technique. Here, we have extended the previous studies to a higher band. In particular, for both $S$ and $P$ waves, we have derived the analytic expressions for the absorption power of a single metal cylinder under an arbitrary external field. These analytic expressions enable us to explain various absorption behaviors of metallic PBG materials using a coherent picture. They explain why $S$ and $P$ waves have the opposite frequency dependence in the absorption coefficient for a fixed structure scale and the decrease of absorption with the square root of the structure scale for a fixed dimensionless frequency. The discussions and conclusions presented here can be generalized to 3D metallic PBG systems as well. Our physical system is described in Sec. II. The results of the transmission and absorption calculations are given in Sec. III and Sec. IV, respectively. A conclusion is given in Sec. V.

\section{PHYSICAL SYSTEMS}

The system we consider here is a square array of $N$ identical metallic cylinders of radius $R$ and dielectric constant $\epsilon$ parallel to the $z$ axis in the air background. We denote $n_{x}$ and $n_{y}$ as the numbers of cylinders in the $x$ and $y$ directions, respectively, and $a$ as the lattice constant.

For metal cylinders, we use the Drude model for the conductivity $\sigma$, i.e., at frequency $f=\omega / 2 \pi, \sigma=\sigma_{0} /(1-i f / \gamma)$, where $\sigma_{0}$ and $\gamma$ are, respectively, the static conductivity and absorption constant of the metal. In terms of the plasma frequency $f_{p}, \sigma_{0}$ becomes $f_{p}^{2} / 2 \gamma$, and the frequency-dependent complex dielectric constant takes the form

$$
\epsilon=\epsilon^{\prime}+\mathrm{i} \epsilon^{\prime \prime}=1-\frac{f_{p}^{2}}{f(f+i \gamma)} .
$$

For all numerical calculations carried out in this work, following Ref. 5, we have chosen $f_{p}=3600 \mathrm{THz}$ and $\gamma$ $=340 \mathrm{THz}$, which corresponds to a conductivity close to that of Ti. However, our discussions and conclusions given below apply to other metal parameters as well.

\section{TRANSMISSION CALCULATIONS}

To calculate the transmission coefficient of a PBG sample, we use the multiple-scattering method. ${ }^{10,11}$ Here we use the formulas derived in Ref. 10. Let the location of the $j$ th cylinder be $\vec{\rho}_{j}=\left(\rho_{j}, \theta_{j}\right)$, where $(\rho, \theta)$ specifies a vector in the $x-y$ plane. We use $u$ to denote the electric (or magnetic) field in the case of $S$ (or $P$ ) waves. When an incident field $u_{\text {in }}(\vec{\rho})$ with a frequency $f$ is applied to the system, the total field $u(\vec{\rho})$ can be written as the sum of the incident field 
and the multiply scattered field arising from all cylinders, i.e., ${ }^{10}$

$$
\begin{aligned}
u(\vec{\rho}) & =u_{\text {in }}(\vec{\rho})+u_{\text {scatt }}(\vec{\rho}) \\
& =u_{\text {in }}(\vec{\rho})+\sum_{j=1}^{N} \sum_{m=-M}^{M} B_{m}(j) H_{m}\left(k_{0} \rho_{\rho j}\right) e^{\mathrm{i} m \theta_{\rho j}},
\end{aligned}
$$

where $k_{0}=2 \pi f / c, \vec{\rho}_{\rho j}=\vec{\rho}-\vec{\rho}_{j}=\left(\rho_{\rho j}, \theta_{\rho j}\right), M=\infty$, and $H_{m}$ is the Hankel function of the first kind. The radiation coefficients $B_{m}(i)$ for any cylinder $i$ are determined by the following self-consistent equations:

$$
\begin{aligned}
B_{m}(i)= & \alpha_{m}^{(0)}(i) D_{m}+D_{m} \sum_{j \neq i} \sum_{l=-M}^{M} B_{l}(j) e^{\mathrm{i}(l-m)\left(\theta_{i j}+\pi\right)} H_{l-m}^{(1)} \\
& \times\left(k_{0} \rho_{i j}\right),
\end{aligned}
$$

for $i=1,2, \ldots, N$, where $\vec{\rho}_{i j}=\vec{\rho}_{j}-\vec{\rho}_{i}=\left(\rho_{i j}, \theta_{i j}\right) . \alpha_{m}^{(0)}(i)$ denotes the expansion coefficients of the incident wave at the $i$ th cylinder, i.e.,

$$
u_{\text {in }}=\sum_{m} \alpha_{m}^{(0)}(i) J_{m}\left(k_{0} \rho_{\rho i}\right) e^{\mathrm{i} m \theta_{\rho i}},
$$

where $J_{m}$ is the Bessel function of first kind. $D_{m}$ can be obtained from the boundary conditions on the surface of a cylinder and has the forms ${ }^{12}$

$$
D_{m}= \begin{cases}\frac{k J_{m}\left(k_{0} R\right) J_{m}^{\prime}(k R)-k_{0} J_{m}^{\prime}\left(k_{0} R\right) J_{m}(k R)}{k_{0} J_{m}(k R) H_{m}^{\prime}\left(k_{0} R\right)-k H_{m}\left(k_{0} R\right) J_{m}^{\prime}(k R)} & \text { for } S \text { waves, } \\ \frac{k_{0} J_{m}\left(k_{0} R\right) J_{m}^{\prime}(k R)-k J_{m}^{\prime}\left(k_{0} R\right) J_{m}(k R)}{k J_{m}(k R) H_{m}^{\prime}\left(k_{0} R\right)-k_{0} H_{m}\left(k_{0} R\right) J_{m}^{\prime}(k R)} & \text { for } P \text { waves, }\end{cases}
$$

where $k=k_{0} \sqrt{\epsilon}$. From the solution of Eq. (3), we can calculate the total scattering amplitude from the expression ${ }^{10}$

$$
f_{s}(\theta)=\sqrt{\frac{2}{\pi k_{0}}} e^{-\mathrm{i} \pi / 4} \sum_{j=1}^{N} \sum_{m=-M}^{M} e^{-\mathrm{i} k_{0} \rho_{i} \cos \left(\theta_{i}-\theta\right)}(-\mathrm{i})^{m} e^{\mathrm{i} m \theta} B_{m}(j) .
$$

From the total field in Eq. (2), the Poynting vector at each point in a $2 \mathrm{D}$ space can also be obtained from

$$
\vec{S}(\vec{\rho})=-\frac{c}{8 \pi k_{0}} \operatorname{Im}\left[u(\vec{\rho}) \nabla u^{*}(\vec{\rho})\right] .
$$

For the transmission calculations, the source beam is prepared by passing a plane wave $u_{0} \exp \left(i k_{0} x\right)$ in the $x$ direction through an open slit of width $w$ in the $y$ direction at a distance $L$ away from the front surface of the PBG sample. ${ }^{10}$ The position of the slit in the $y$ direction lies in the middle of the sample. In all the calculations below, we have set $u_{0}$ $=1$ as the units of electric (or magnetic) field. With this configuration, the transmission coefficient can be calculated from the expression ${ }^{10}$

$$
T=\left|1+\sqrt{\frac{2 \pi}{k_{0} w^{2}}} e^{\mathrm{i} \pi / 4} f_{s}(0)\right|^{2} .
$$

In our numerical calculations for $S$ waves, we have chosen a five-layer sample $\left(n_{x}=5\right)$ with a width $n_{y}=50$. The values for $w$ and $L$ are 20 and 21, respectively, in units of the lattice constant $a$. The results of our calculations are insensitive to the values of $w$ and $L$. For all the calculations below, we have fixed the ratio $R / a=0.1784$, which gives a filling ratio 0.1 . The results of transmission coefficient $T(\hat{f})$ as a function of dimensionless frequency $\hat{f} \equiv f a / c$ are plotted in curves $A, B$, and $C$ of Fig. 1 for $a=1.27,0.127$, and 0.0127 $\mathrm{mm}$, respectively. Our calculations converge when $M \geqslant 2$.
Both the position of the first gap below the cutoff frequency at $\hat{f}_{c} \approx 0.5$ and the position of the second gap from $\hat{f} \approx 0.60$ to 0.84 seem to be insensitive to the structure scale $a$ even in the presence of large absorption. This is true as long as the frequency $f$ is much smaller than the absorption constant $\gamma$ so that the dielectric constant can be approximated by

$$
\epsilon^{\prime} \approx 1-f_{p}^{2} / \gamma^{2} \approx-111, \quad \epsilon^{\prime \prime} \approx f_{p}^{2} / f \gamma \approx 38117 / f(\mathrm{THz}) .
$$

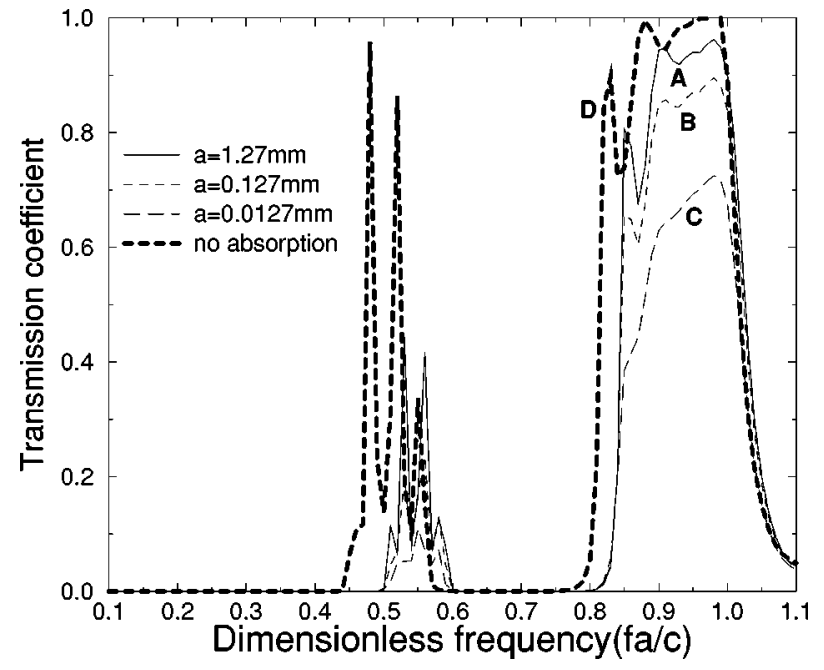

FIG. 1. Transmission coefficient for $S$ waves with the lattice constant $a=1.27(A), 0.127(B)$, and $0.0127 \mathrm{~mm}(C)$. Curve $D$ is obtained by ignoring the absorption. 


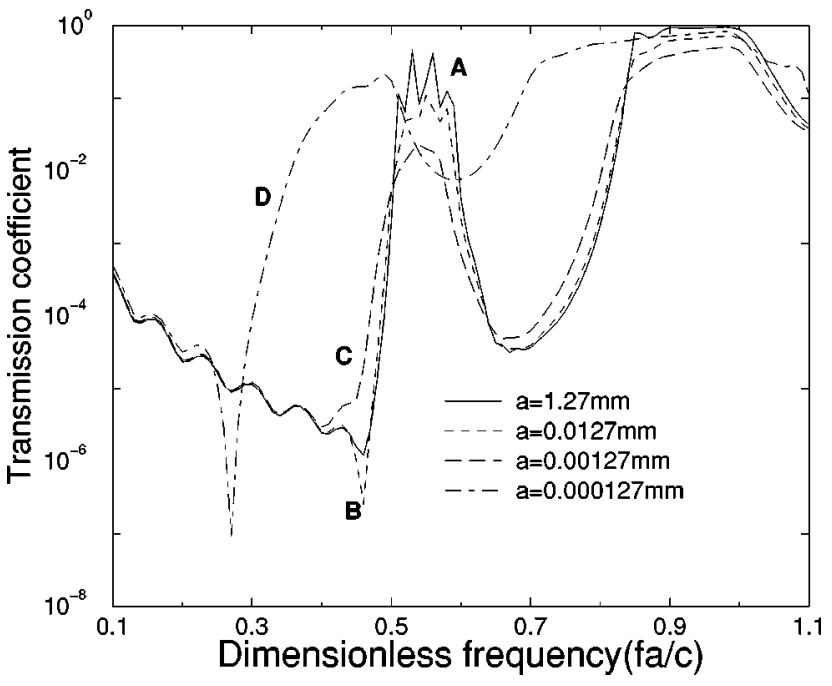

FIG. 2. The logarithm of the transmission coefficient for $S$ waves with the lattice constant $a=1.27$ (A), $0.0127(B), 0.00127$ $(C)$, and $0.000127 \mathrm{~mm}(D)$.

It is easy to see that Eq. (9) implies $\epsilon^{\prime \prime} \gg 111$. The maximum frequency shown in Fig. 1 is about $f \approx 30 \mathrm{THz}$, which occurs when $\hat{f} \approx 1$ and $a=0.0127 \mathrm{~mm}$. This is still one order of magnitude smaller than $\gamma$. Thus, in the units of $\hat{f}$, the location of a gap remains unchanged even with two orders of magnitude variation in $\epsilon^{\prime \prime}$. To see how the presence of absorption affects the gap position, we also plot in curve $D$ of Fig. 1 the transmission result for the case of $\epsilon^{\prime}=-111$ and $\epsilon^{\prime \prime}=0$. Compared to curves $A, B$, and $C$, significant shifts in the gap positions can be observed. To approach the limit of perfect conductors, we have done a similar calculation with $\epsilon^{\prime}=-1000$, and found no further changes in the gap positions. Thus, these results indicate that a gap position determined from perfect conductors may produce some shift from that of real metals.

When the frequency region is on the order of $\gamma$ or above, the gap position starts to deviate from the scaling behavior shown in Fig. 1. In Fig. 2, we plot the logarithm of $T(\hat{f})$ for the cases of $a=0.127,0.0127,0.00127$, and $0.000127 \mathrm{~mm}$ in curves $A, B, C$, and $D$, respectively. From curves $A$ and $B$, we found that the quality of the gap is also insensitive to the structure scale as long as Eq. (9) is valid. This is due to the small absorption that occurred inside the gaps. This scaling is no longer true for curves $C$ and $D$, when the frequency is close to or above the value of $\gamma$. For the case of $P$ waves, we have chosen $n_{x}=10, n_{y}=30, w=10 a$, and $L=18 a$. The results of $T(\hat{f})$ for the cases of $a=1.27,0.127$, and 0.00127 $\mathrm{mm}$ are plotted as curves $A, B$, and $C$ in Fig. 3, respectively. Again, the gap positions seem to be invariant (in units of $\hat{f}$ ) for all structure scales except that some deviation occurs in the second gap of curve $D$ where the frequency $(f$ $=300 \mathrm{THz}$ ) is close to $\gamma=340 \mathrm{THz}$. Also plotted in Fig. 3 are the heavy dots near the band gaps obtained by ignoring the absorption. A shift in the band-gap position is also seen in both gaps.

\section{ABSORPTION CALCULATIONS}

From the transmission curves $A, B$, and $C$ shown in Fig. 1, it is easy to see that, for $S$ waves, the amount of absorption in

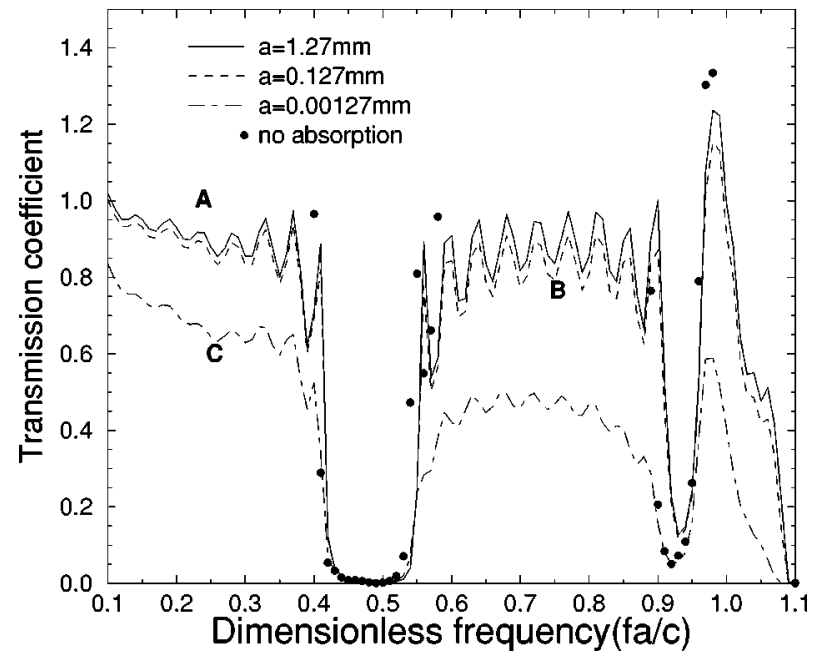

FIG. 3. Transmission coefficient for $P$ waves with the lattice constant $a=1.27(A), 0.127(B)$, and $0.00127 \mathrm{~mm}(C)$. The heavy dots are the result obtained by ignoring the absorption.

the first two bands increases with decreasing frequency for a fixed structure scale $a$. For the case of $P$ waves, the results of Fig. 3 seem to indicate the opposite, i.e., the amount of absorption in the first two bands increases with increasing frequency for a fixed value of $a$. However, for both $S$ and $P$ waves, the amount of absorption decreases with increasing structure scale $a$ for a fixed value of $\hat{f}$.

To see these behaviors more explicitly, we have also calculated the absorption power per unit length in the $z$ direction, $P_{a}$, by performing a contour intergral of the Poynting vector along any closed loop $C$ in the 2D plane enclosing the PBG sample, i.e., $\oint_{C} \vec{S}(\vec{\rho}) d \vec{n}$, where $\vec{n}$ denotes the unit vector normal to loop $C$. Here we define the absorption coefficient $A$ as the ratio of absorption power to incident power $I_{p}=c w / 8 \pi$, i.e., $A=P_{a} / I_{p}$. For $S$ waves, the results of $A(\hat{f})$ are shown in curves $A, B$, and $C$ of Fig. 4 for structure scales $a=1.27,0.127$, and $0.0127 \mathrm{~mm}$, respectively. The corresponding results for $P$ waves are plotted in Fig. 5. As a function of frequency, the absorption behaviors found in the first two bands are consistent with those seen from the trans-

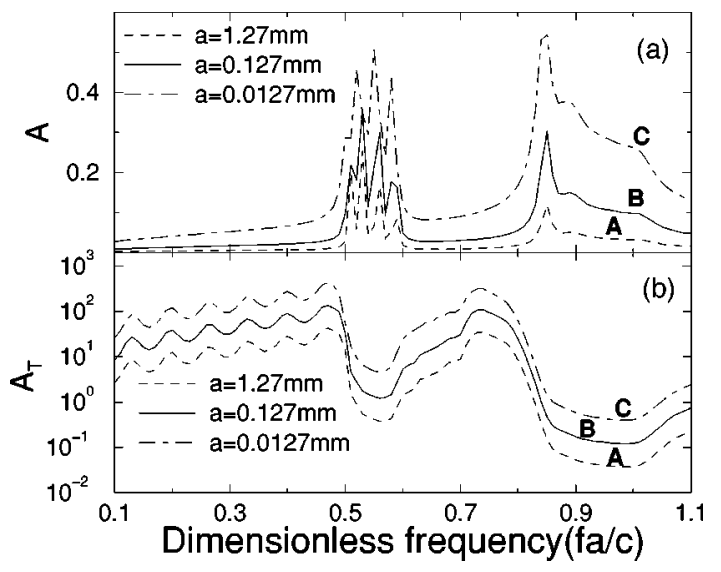

FIG. 4. (a) The absorption coefficient for $S$ waves with the lattice constant $a=1.27(A), 0.127(B)$, and $0.0127 \mathrm{~mm}(C)$. (b) Replotting of (a) using the ratio of the absorption power to the transmitted power. 


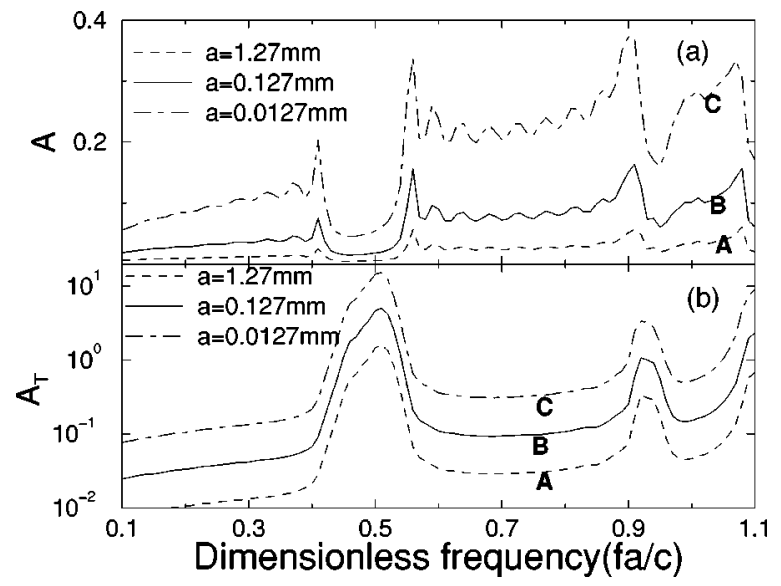

FIG. 5. (a) The absorption coefficient for $P$ waves with the lattice constant $a=1.27(A), 0.127(B)$, and $0.0127 \mathrm{~mm}(C)$. (b) Replotting of (a) using the ratio of the absorption power to the transmitted power.

mission results. The reduction of absorption coefficient inside a gap is due to the exponential decay of the wave intensity from the front surface of the PBG samples. Also, for both $S$ and $P$ waves, the absorption coefficient seems to decrease with $\sqrt{a}$ for a fixed $\hat{f}$. This square root dependence was first pointed out in Ref. 5, where the authors interpreted this by using the ratio of the skin depth to the structure scale, i.e., $\delta / a \sim \sqrt{a}$.

In order to have a complete understanding of all the absorption behaviors found above, we would like first to study the absorption behavior of a single cylinder. For both $S$ and $P$ waves, we have derived the analytic expressions for the absorption power of a single metal cylinder in an arbitrary applied field. A brief derivation is outlined in the Appendix. Here we are only interested in the frequency region $f \ll \gamma$ where Eq. (9) is valid. The results for the absorption power per unit length in the $z$ direction are

$$
\bar{P}_{a} \simeq \begin{cases}\frac{2 \sigma_{0} R^{2}}{\pi|k R|^{2} \operatorname{Im}(k R)} \sum_{m} \frac{\left|\alpha_{m}\right|^{2}}{\left|H_{m}\left(k_{0} R\right)\right|^{2}} & \text { for } S \text { waves, } \\ \frac{2 \sigma_{0} R^{2}}{\pi|k R|^{2} \operatorname{Im}(k R)} \sum_{m} \frac{\left|\alpha_{m}\right|^{2}}{\left|H_{m}^{\prime}\left(k_{0} R\right)\right|^{2}} & \text { for } P \text { waves, }\end{cases}
$$

where $\alpha_{m}$ represents the expansion coefficients of the applied field at the center of the cylinder, i.e.,

$$
\tilde{u}_{\mathrm{in}}=\sum_{m} \alpha_{m} J_{m}\left(k_{0} \rho\right) e^{\mathrm{i} m \theta} .
$$

The difference between $S$ and $P$ waves in the absorption power lies in the different frequency dependence of the electric field on the surface of the metal cylinder. In fact, in the limit of large $R$ and a plane-wave incidence, Eq. (10) reduces to the well-known absorption result of an infinite metallic interface. To see this, let us consider the case when the cylinder radius $R$ and the slit width of the applied field satisfy the inequality $2 \pi / k_{0} \ll w<R$. Due to its finite beam width, the incident wave can only excite the cylinder below a maximum angular momentum number $k_{0} w / 2$. Thus, we can ap-

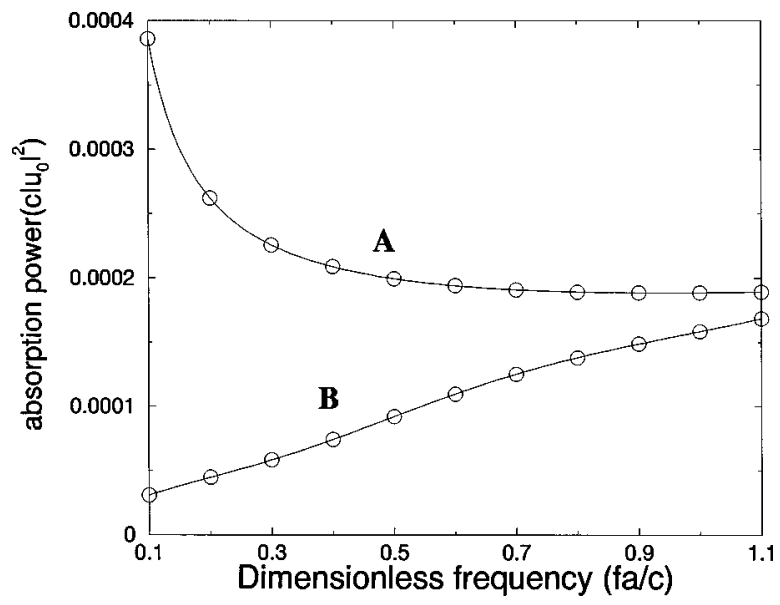

FIG. 6. For a single metal cylinder in a plane-wave incidence of strength $u_{0}$, the absorption power per unit length in the $z$ direction is plotted as a function of dimensionless frequency $\mathrm{fa} / \mathrm{c}$ in units of c $\left|u_{0}\right|^{2}$. Curves $A$ and $B$ are the analytic results for $S$ and $P$ waves, respectively. The open circles are the corresponding numerical data obtained from the total field in Eq. (2).

proximate $\alpha_{m}$ by using the expansion of a plane wave $\exp \left(i k_{0} x\right)$, i.e., $\mathrm{i}^{m}$ when $|m|<k_{0} w / 2$ and take $\alpha_{m} \approx 0$ when $|m|>k_{0} w / 2$. Since $k_{0} R \gg 1$, we can also approximate both $\left|H_{m}\left(k_{0} R\right)\right|^{2}$ and $\left|H_{m}^{\prime}\left(k_{0} R\right)\right|^{2}$ by $2 / \pi k_{0} R$. Furthermore, if we take $|k|^{2}=\left|k_{0}\right|^{2}|\epsilon| \approx 2 \sigma_{0} / f$ and relate $\operatorname{Im} k$ to the skin depth, i.e., $\operatorname{Im} k \approx 1 / \delta=2 \pi \sqrt{f \sigma_{0}} / c$, both expressions in Eq. (10) converge to $\bar{P}_{a} \sim f \delta w / 2$. The frequency-dependent part $f \delta$ in $\bar{P}_{a} / w$ represents nothing but the absorption per unit area of a normally incident wave on an infinite metal surface, i.e., $\delta|\vec{E}|^{2}$, and the factor $f$ arises from the $\sqrt{f}$ dependence of the electric field $|\vec{E}|$ on the metal surface. ${ }^{13}$

When the wavelength becomes comparable to $R$ as in the case of PBG samples, the condition $k_{0} R \gg 1$ is no longer true and higher-order terms of $\left|H_{m}\left(k_{0} R\right)\right|^{2}$ and $\left|H_{m}^{\prime}\left(k_{0} R\right)\right|^{2}$ in Eq. (10) contribute in different ways. This gives rise to different frequency dependence for $S$ and $P$ waves. In order to demonstrate this and to compare with the results shown in Figs. 4 and 5, we take a cylinder of radius $R=0.1784 a$ with $a=1.27 \mathrm{~mm}$ and plot the analytic results of $\bar{P}_{a} / c$ in Eq. (10) from a plane-wave incidence, i.e., $u_{\text {in }}=\exp \left(i k_{0} x\right)$, as functions of $f a / c$ by two solid curves in Fig. 6. In order to check Eq. (10) independently, we have also performed numerical calculations of the absorption power directly by using the total field obtained from the scattering of a single cylinder given in Eq. (2). These numerical results are shown by open circles in Fig. 6, which coincide exactly with the analytical results. The opposite frequency dependence in the absorption power for $S$ and $P$ waves shown in Fig. 6 explains the similar behaviors found in the absorption coefficient of the first two bands in Figs. 4(a) and 5(a). In the limit of large $\hat{f}$, the convergence of two curves indicates that the difference in the absorption behavior for $S$ and $P$ waves will diminish in higher bands. In Figs. 4(a) and 5(a), the fluctuations of $A(\hat{f})$ inside a pass band arise from the fluctuations in the reflection coefficient due to the finite thickness of PBG samples. In order to filter out these fluctuations, we normalize the absorption power by the transmitted power $T_{p}$ instead 
of the incident power, i.e., $A_{T}=P_{a} / T_{p}$. The results of $A_{T}$ are plotted in the logarithmic scale in Figs. 4(b) and 5(b) for $S$ and $P$ waves, respectively. For frequencies inside the bands, $A_{T}$ becomes a smooth function of $\hat{f}$, and $S$ and $P$ waves have clearly the opposite frequency dependence in absorption.

From Figs. 4(b) and 5(b), we also see clearly that all curves are separated roughly by a constant $\sqrt{10}$. This implies that $A_{T}$ has a $1 / \sqrt{a}$ dependence on the structure scale for a fixed $\hat{f}$. To understand this, we again refer to the analytic expressions of the absorption in a single metal cylinder. Since $\hat{f}$ and $R / a$ are both fixed, the summations in Eq. (10) become invariants. The common factor in front of them is proportional to $\sqrt{R} /(f R)^{3 / 2}$. For the case of PBG samples, we can naively assume that all the cylinders have the same absorption power for frequencies inside a pass band. From the definition of the absorption coefficient, we have $A(f)$ $\approx n_{x} n_{y} \bar{P}_{a} /(c w / 8 \pi)$. Since $\bar{P}_{a}$ depends on the structure scale as $\sqrt{a}$ and the slit width $w$ is proportional to $a$, we obtain a $1 / \sqrt{a}$ dependence in the absorption coefficient.

\section{CONCLUSIONS}

We have carried out a systematic study on the transmission and the absorption behaviors of 2D metallic PBG materials. We find that, in the frequency region $f \ll \gamma$, the position of a gap becomes invariant in units of $\hat{f}$ even with the presence of absorption. By ignoring the imaginary part of the dielectric constant for the metallic component, we find a significant shift in the gap position. We also find that the absorption behaviors of a metallic PBG sample can be understood by those of a single metal cylinder. For a fixed structure scale $a$, the absorption coefficent in the bands decreases with the frequency for the case of $S$ waves. An opposite frequency dependence is found for the case of $P$ waves. At a given $\hat{f}$, the absorption coefficient is found to scale with $1 / \sqrt{a}$. The relationship between the absorption behavior of a PBG sample and that of a single metal cylinder can be generalized to 3D systems as well.

\section{ACKNOWLEDGMENTS}

The authors wish to thank C. T. Chan and K. M. Ho for useful discussions. The work was supported by Hong Kong RGC Grant No. HKUST 6137/97P.

\section{APPENDIX: THE ABSORPTION POWER IN A SINGLE METAL CYLINDER}

In the frequency range where Eq. (9) is valid, the power absorbed by a metal cylinder per unit length in the $z$ direction is given by

$$
\bar{P}_{a}=\left(\sigma_{0} / 2\right) \int_{m} d \vec{\rho} \vec{E} \cdot \vec{E}^{*}
$$

where $\sigma_{0}$ is the static conductivity and the subscript " $m$ '" denotes that the integration is carried out inside the metal cylinder only. The electric field $\vec{E}$ in Eq. (A1) takes the form

$$
\vec{E}= \begin{cases}u \mathbf{e}_{z} & \text { for } S \text { waves, } \\ -\frac{i}{\epsilon k_{0}} \nabla u & \text { for } P \text { waves, }\end{cases}
$$

where $\mathbf{e}_{z}$ is the unit vector in the $z$ axis and the gradient is taken in the $x-y$ plane. Under an arbitrary applied field of Eq. (11), the field inside the metal cylinder can be written in the form

$$
u=\sum_{m} c_{m} J_{m}\left(k_{0} \rho\right) e^{\mathrm{i} m \theta} .
$$

The coefficient $c_{m}$ should be determined by the boundary conditions on the surface of the cylinder and has the form

$$
c_{m}=\alpha_{m} \frac{2 \mathrm{i}}{\pi R \Delta_{m}},
$$

where

$$
\Delta_{m}= \begin{cases}k_{0} J_{m}(k R) H_{m}^{(1)^{\prime}}\left(k_{0} R\right)-k J_{m}^{\prime}(k R) H_{m}^{(1)}\left(k_{0} R\right) & \text { for } S \text { waves, } \\ k_{0} J_{m}(k R) H_{m}^{(1)^{\prime}}\left(k_{0} R\right)-(k / \epsilon) J_{m}^{\prime}(k R) H_{m}^{(1)}\left(k_{0} R\right) & \text { for } P \text { waves. }\end{cases}
$$

If we assume that the radius of the metal cylinder is much greater than the skin depth $\delta$, i.e., $\operatorname{Im} k R \gg 1$, the following asymptotic limits become valid:

$$
J_{m}(k R) \rightarrow \mathrm{i}^{m} e^{\mathrm{i} \pi / 4} e^{-\mathrm{i} k R} / \sqrt{2 \pi k R}, \quad J_{m}^{\prime}(k R) \rightarrow \mathrm{i}^{m-1} e^{\mathrm{i} \pi / 4} e^{-\mathrm{i} k R} / \sqrt{2 \pi k R} .
$$

By subsitituting Eqs. (A2)-(A6) into Eq. (A1), with some straightforward manipulations, we finally obtain Eq. (10).

*Permanent address: Department of Modern Applied Physics, Tsinghua University, Beijing 100084, China.

${ }^{1}$ For example, see Photonic Band Gap Materials, edited by C. M. Soukoulis (Kluwer Academic, Dordrecht, 1996).

${ }^{2}$ J. D. Joannopoulos, R. D. Meade, and J. N. Winn, Photonic Crys- tals Molding the Flow of Light (Princeton University Press, Princeton, NJ, 1995).

${ }^{3}$ A. R. McGurn and A. A. Maradudin, Phys. Rev. B 48, 17576 (1993).

${ }^{4}$ D. R. Smith, S. Schultz, N. Kroll, M. Sigalas, K. M. Ho, and C. 
M. Soukoulis, Appl. Phys. Lett. 65, 645 (1994).

${ }^{5}$ M. M. Sigalas, C. T. Chan, K. M. Ho, and C. M. Soukoulis, Phys. Rev. B 52, 11744 (1995).

${ }^{6}$ N. A. Nicorovici, R. C. McPhedran, and L. C. Botten, Phys. Rev. E 52, 1135 (1995).

${ }^{7}$ S. Fan, P. R. Villeneuve, and J. D. Joannopoulos, Phys. Rev. B 54, 11245 (1996).

${ }^{8}$ M. Stoytchev and A. Z. Genack, Phys. Rev. B 55, R8617 (1997).
${ }^{9}$ D. F. Sievenpiper, E. Yablonovitch, J. N. Winn, S. Fan, P. R. Villeneuve, and J. D. Joannopoulos, Phys. Rev. Lett. 80, 2829 (1998).

${ }^{10}$ L. M. Li and Z. Q. Zhang, Phys. Rev. B 58, 9587 (1998).

${ }^{11}$ G. Tayeb and D. Maystre, J. Opt. Soc. Am. A 14, 3323 (1997).

${ }^{12}$ H. C. van de Hulst, Light Scattering by Small Particles (Dover, New York, 1981).

${ }^{13}$ For example, J. D. Jacson, Classical Electrodynamics, 2nd ed. (John Wiley \& Sons, New York, 1975). 